\title{
Evaluation of Comprehensive Utilization of Land of Jiangzhehu in China Based on RR-GASVM
}

\author{
Li Chen*
}

School of Management, Anhui Jianzhu University, Hefei, Anhui, China

\begin{abstract}
This paper established evaluation index system of comprehensive utilization of land from the three aspects of economic benefits, ecological benefits and social benefits. Firstly, ridge regression (RR) is used to the indicator index system for screening, and then genetic algorithm and support vector machine (that is GASVM) is trained to the transformed samples. Moreover, the comprehensive evaluation results between the GASVM and ridge regression and genetic algorithm and support vector machine(that is RR-GASVM) is compared, which results by MATLAB show that using RRGASVM is more accurate and higher efficient for the comprehensive utilization of land evaluation than that of GASVM. The combination of ridge regression and genetic algorithm and support vector machine can improve the decision ability of SVM, so that the decision has higher adaptability, which is the development direction of future research. At last, based on the results of this study, some suggestions are put forward accordingly. The area of Jiangsu, Zhejiang and Shanghai (that is Jiangzhehu) should consolidate the existing achievements and strengthen the innovation of the land management system on the basis of utilizing land comprehensively, realize the intensive, large-scale operation and speed up agricultural modernization development by accelerating rural land circulation, and improve the efficiency of land utilization in order to save the land of urban construction. It is clear that people should insist the principle of paying equal attention to development and consolidation, and strengthen the cultivated land's ability to resist natural disasters in order to improve the ecological environment at the same time that improved the land utilization.
\end{abstract}

Keywords: Comprehensive utilization of land, evaluation, GA - SVM, ridge regression.

\section{INTRODUCTION}

With the acceleration of new urbanization development in China, the land scope and intensity which people used is increasing and the development of urban land appeared the trend of extensive expansion in our country. Land resources, especially cultivated land resources are reducing sharply. The contradiction of population and land is growing, that the urbanization of land is faster than that of population and the utilization of construction land is extensive and inefficient. The faster growth of economy and population, the pressure of human activities on the environment is increasing day by day, at the same time, the problem of land pollution, land desertification, the soil erosion become serious and prominent. In the situation of very limited land resources, the rational utilization of land resources has become a hot topic.

There are some people who put forward to go the road of urbanization and intensive use of land, such as Wang Yong and Yang xiaoyu [1], Yang Fan [2], and they stressed that the intensive utilization of land is an important guarantee for the healthy development of urbanization. QU Li-ping et al. [3] analyzed the reasons which restrict the intensive utilization of city land in China. Wang Aimin [4] pointed out the problem and difficulties of the intensive utilization of urban land. Qiu Lei et al. [5] evaluated the intensive degree of utilization of 10 cities by combining entropy value method comprehensively.

Wang Wengang, et al. [6] measured the efficiency of land utilization of 31 provinces in Chinese mainland by using DEA method. Li Huan et al. [7] evaluated level of intensive land utilization in development area of 11 cities in Zhejiang province by BP artificial neural network. Wu Jia [8] constructed the evaluation model of city land intensive utilization based on AHP from the structure of city land utilization, the intensity of city land investment, the degree of urban land utilization and benefits of urban land utilization.

Ming Hua, et al. [9] established the evaluation index system of city land intensive utilization level by using principal component analysis to evaluation of the city of Xining city's land intensive utilization level method from three aspects, the degree of land utilization and the efficiency of land utilization.

With the increasing of influencing factors, the dimension of the evaluation system evaluation increase in the process of establish model in the comprehensive evaluation of land, which will become the problem of high dimension. In the analysis, DEA, analytic hierarchy process, artificial neural network method, entropy method are difficult to meet the requirements of high dimension. In the solving of the high dimension problem, the advantage of SVM is recognized by scholars. There are perfect theory basis in SVM, it not only 
eliminates the neural network is affected by the complexity of network structure and the big sample capacity, prone to appear the symptom of study excessively and low generalization ability, but also has high generalization ability. In this paper, the evaluation methods of land's comprehension utilization which combined ridge regression analysiss with the genetic algorithm, support vector machine to evaluate land utilization in Jiangzhehu.

This paper is organized as follows. First, international and national knowledge of comprehensive utilization of land. Second, material and methods are put forward which include overview of the study area and comprehensive evaluation of land data feature selection and extraction based on the ridge regression and the achievement of land intensive evaluation based on RR-GASVM. In section 2, the objective comprehensive evaluation index system of land utilization in Zhejianghu is also established. Third, the comprehensive evaluation results of Jiangzhehu between the GASVM and RRGASVM is compared. Finally, the article gives conclusion and discussion.

\section{MATERIAL AND METHODS}

\subsection{Material of the Study Area}

Jiangsu is located in the downstream of Yangtze River and Huaihe River, the shore of East Sea and Yellow Sea, and is an important part of the Yangtze River Delta region, one of the provinces of the highest population density in the China. The total area is $102600 \mathrm{~m}^{2}$, accounting for $1.11 \%$ of the total area, ranking twenty-fourth in the nation and the land of per person is least in the all provinces. The plain and water area account for $69 \%$ and $17 \%$, that proportion is the first in our country. The terrain in Jiangsu province is located in plain, Jianghuai plain, plain area of 70000 square kilometers, accounting for more than $70 \%$ of the province's area, and is mainly composed of South of Jiangsu plain, the Soviet Union in the northern Jiangsu Plain, Jianghuai plain and Huanghuai plain. Low mountains and hills located in the north and southwest accounted for $14.3 \%$ of the total area in the province.

Zhejiang is located in the south wing of Yangtze River which land area is 101800 square kilometers. Zhejiang province is located in the east of the East China Sea, south of Fujian, connected with the Jiangxi, Anhui in the west, and close to Shanghai and Jiangsu in the north. The distance of Jiangsu between south and north is about 450 kilometers and land area is 101800 square kilometers, that $1.06 \%$ of the country. Zhejiang is one of the smallest provinces in China. There is complex terrain in Zhejiang, mountains and hills accounting for $70.4 \%$, plains and basins occupied $23.2 \%$.

Shanghai is a part of the alluvial plain of the Yangtze River Delta, There is almost plain that is low and within many rivers except few hills in the southwestern. Shanghai is close to the Yangtze River in the north which is near to Hangzhou Bay in the south and meets Jiangsu and Zhejiang provinces in the west. This area is 6340.5 square kilometers in total, of which the area of land is 5643.5 square kilometers and the water area is 697 square kilometers. The coastline is about 172 kilometers.

\subsection{Methods}

\subsubsection{Comprehensive Evaluation of Land Data Feature Selection and Extraction Based on the Ridge Regression (RR)}

In 1962, American statistician Arther Hoerl proposed the ridge regression method firstly. The main principle is that qualitative analysis was carried out on the dependent variable $\mathrm{Y}$, and determines the regression factor, the independent variable.

When there is multiple linearity among the independent variables, namely

$\mathrm{X}^{\mathrm{T}} \mathrm{X} \mid=0$

Through the introduction of a small ridge parameter $\mathrm{K}$ in the correlation matrix $(1>\mathrm{K}>0)$, add it to the main diagonal elements in order to reduce the influence of the least square estimation of parameter double common liner feature vector and added it to the $\mathrm{X}^{\mathrm{T}} \mathrm{X}$ of a normal matrix $\mathrm{kI}(\mathrm{k}>0$, I as a unit matrix).

Finally, got the estimator based on ridge regression:

$\beta(\mathrm{k})=\left(\mathrm{X}^{\mathrm{T}} \mathrm{X}+\mathrm{kI}\right)^{-1} \mathrm{X}^{\mathrm{T}} \mathrm{Y}$

Obviously, when $\mathrm{k}=0$, the estimator of ridge regression is the least squares estimation, when $\mathrm{k}$ approaches infinity, the estimator of ridge regression tends to 0 , so $\mathrm{K}$ should not be too large. To determine ridge parameter values by selecting the appropriate method and calculate the ridge regression results when $\mathrm{K}$ is different. The biggest advantage of ridge regression analysis method is to eliminate the influence of multiple common linearity and the estimation results are more stable and effective than the multiple linear regression of least square method and regression step by step. Ridge regression can solve the problem of redundant information between features, and access easily to the information of data classification [10].

In the data of the evaluation land's intensive utilization, the selection and extraction of data feature is an important step. When people don't have the appropriate method to screen indexes, but all data input training, the complexity of the comprehensive evaluation of land will be high. The efficiency of comprehensive evaluation will below and which will affect the accuracy of land's intensive evaluation. To use the ridge regression selection, training, the complexity of the comprehensive evaluation of land would be reduced. RRGASVM evaluation process diagram is such as Fig. (1). The curve of RR-GA-SVM evaluation prediction is such as Fig. (2).

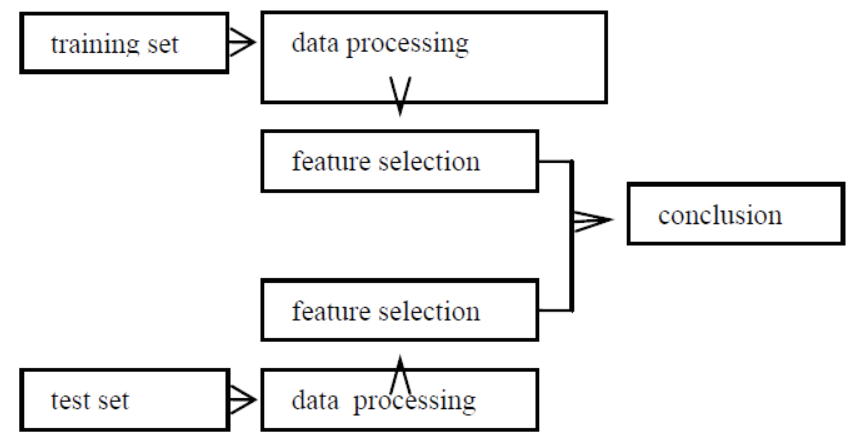

Fig. (1). RR-GASVM evaluation process diagram. 


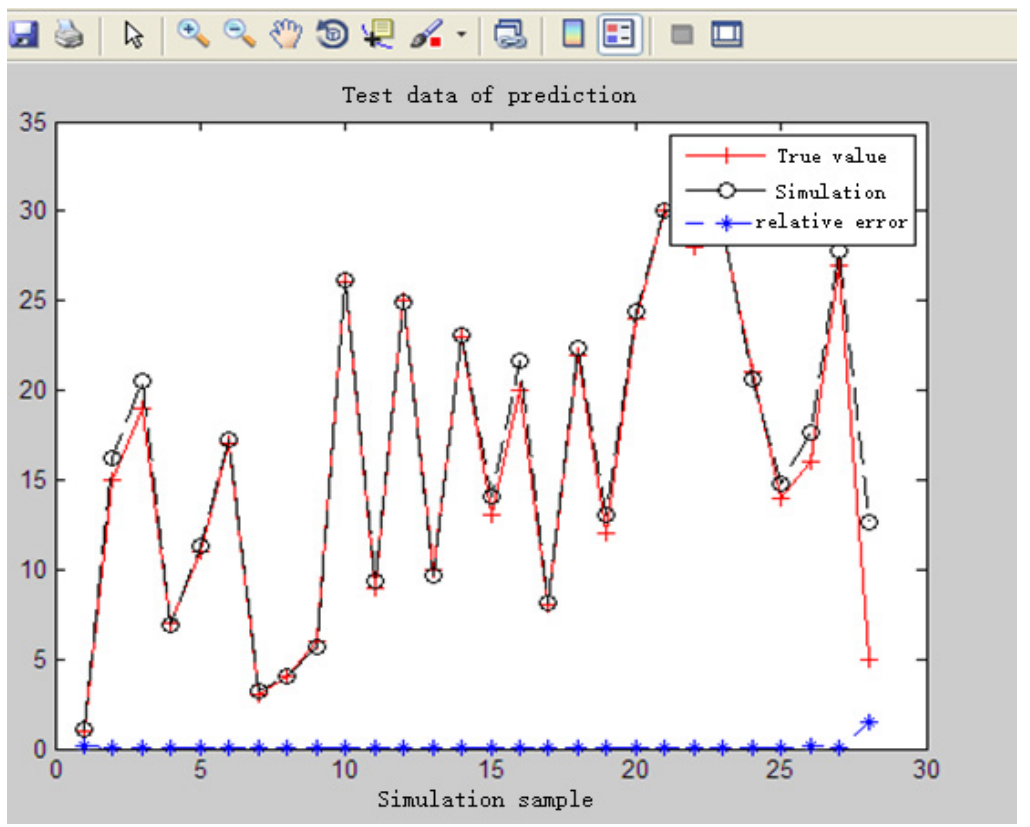

Fig. (2). The curve of RR-GA-SVM evaluation prediction.

\subsubsection{Methods of Land Intensive Evaluation Based on RR- GASVM}

(1) The principle of SVM

The founder of SVM, V. Vapnik began to study the statistical learning theory as early as 60s. In 1971, V. Vapnik and A. Chervonenkis proposed an important theoretical foundation of SVC-VC dimension theory. In 1982, V. Vapnik proposed the principle of the structural risk minimization, which was called the cornerstone of SVM algorithm and of epoch-making significance. In 1995, Cortes and Vapnik further explored the classification of nonlinear optimal boundary, and in 1995, they proposed the classification of SVM completely.

For given training sample set:

$D=\left\{\left(x_{i}, y_{i}\right) \mid x_{i} \in R^{d}, y_{i} \in R\right\}, i=1,2, \ldots, l$

SVM solves linear inseparable in the original space $\mathrm{x}$ by mapping the original variable into a high dimensional space $^{\varphi(\cdot)}$.

In the high dimension space, finding $\omega$ based on structural risk minimization.

Non-negative slack variable and penalty factor $\mathrm{C}$ can be expressed as optimization problems.

$$
\begin{gathered}
\min \left(\frac{1}{2} \omega^{T} \omega+C \sum_{i=1}^{l}\left(\xi_{i}+\xi_{i}^{*}\right)\right) \\
\left\{\begin{array}{c}
y-\omega^{T} \varphi\left(x_{i}\right)-b \leq \varepsilon+\xi_{i} \\
\omega^{T} \varphi\left(x_{i}\right)+b-y \geq \varepsilon+\xi_{i}^{*} \\
\xi_{i}, \xi_{i}^{*} \geq 0
\end{array}\right.
\end{gathered}
$$

Here, $\mathrm{C}$ is a given constant beforehand, and the upper boundary and lower boundary which controlling output constraint.
Lagrange multiplier.

$$
\begin{aligned}
& L=\frac{1}{2} \omega^{T} \omega+C \sum_{i=1}^{l}\left(\xi_{i}+\xi_{i}^{*}\right)-\sum_{i=1}^{l} \alpha_{i}\left(\varepsilon+\xi_{i}-y+\left(\omega^{T} \varphi\left(x_{i}\right)\right)+b\right) \\
& -\sum_{i=1}^{l} \alpha_{i}\left(\varepsilon+\xi_{i}+y-\left(\omega^{T} \varphi\left(x_{i}\right)\right)-b\right)-\sum_{i=1}^{l}\left(\eta_{i} \xi_{i}+\eta_{i}^{*} \xi_{i}^{*}\right)
\end{aligned}
$$

Here, it is though laser multiplier, which is only part of the zero, that will be marked as nonzero though laser product factor, which is the support vector that is obtained by SVM training.

According to the theorem, it is known that appropriate kernel function is used in the optimal

$\mathrm{K}(\mathrm{xi}, \mathrm{yi})=(\Phi(\mathrm{xi}) . \Phi(\mathrm{xi}))$

hyper plane.

At this point, the objective function of two time programming becomes

$w(\alpha)=\sum_{i=1}^{l} \alpha_{l}-\frac{1}{2} \sum_{i=1}^{l} \sum_{j=1}^{l} \alpha_{i} \alpha_{i} y_{i} y_{j} K\left(x_{i} \quad x_{j}\right)$

The corresponding classification decision function also becomes

$f \mathrm{c} x \quad \operatorname{sgn}\left(\sum_{i=1}^{l} \alpha_{i} y_{i} K\left(x_{i} \quad x_{j}\right)+b\right)$

This is the support vector machine [11].

To improve the generalization ability of SVM by the structural risk minimization principle, SVM has a set of visual and good classification ability which linearly inseparable samples of low dimensional space will be mapped to high dimensional feature space by a nonlinear transformation of kernel function and construct the optimal hyper planes and decision function in low dimensional space. According this, people can solve a given $\mathrm{X}$ and find a real valued function 
$\mathrm{f}(\mathrm{x})$ on the training set Rn, and then infer the output $\mathrm{y}$ which the $\mathrm{X}$ correspond. Parameter selection of support vector machines $\mathrm{C}$ and kernel function parameter is a complex problem.

The domestic academic circles optimize the parameters of SVM by the neural network, which has the learning problem, namely, it looks nice in learning results, but the generalization ability is poor in fact. Neural network training input layer nodes and the number of hidden layer nodes is proposed with reference to some experience formulas to determine, which affect the training results. Adaptive genetic algorithm global optimization characteristics, using the characteristics of genetic algorithm (that is GA), support vector machine model is constructed for the adaptive parameter optimization algorithm to get the optimal parameter values of SVM, and effectively improve the accuracy and efficiency of the evaluation, which provides an effective way to solve the problem of selecting the parameters of support vector machine.

(2) The genetic algorithm optimized support the achievement of vector machine (GASVM)

Genetic algorithm is a kind of method to search the optimal solution by simulating the process of natural evolution, it initially by Professor J. Holland Michigan of the University of American first proposed in 1975 which is a kind of search algorithms that can be used in complex system optimization with robustness. Compared with the traditional optimization algorithm, the traditional optimization algorithms tend to deal directly with the actual value of the decision variables, and the genetic algorithm can imitate biological genetic and the genetic algorithm can intimate biological genetic and evolutionary mechanism of nature and regarded fitness as the search information directly, without derivatives and other auxiliary information. Genetic algorithm is used to search for information of multiple points, with implicit parallelism. The genetic algorithm uses probability search technology, and non-deterministic rules [12].

After the processing of comprehensive evaluation of land data, people transmit it to the evaluation in order to achieve the evaluation results of GA-SVM. The comprehensive evaluation method of land based on the RR-GASVM is not that construct plane on the sample directly, but carries out ridge regression transform on the sample, then to use GASVM to transform the training samples in order to get the optimal hyper plane to classify samples. The principle of RR-GASVM is as follows. ple.

Step 1: Input samples and ridge regression on the sam-

Step 2: Generating initial population with random or a certain probability and selecting of a number of individual. data.

Step 3: Forming the corresponding training data and test

Step 4: Training data and test data is used to train and test by SVM according to the penalty factor $\mathrm{C}$ of each chromosome and kernel function value.

Step 5: Calculating the fitness value of each individual.

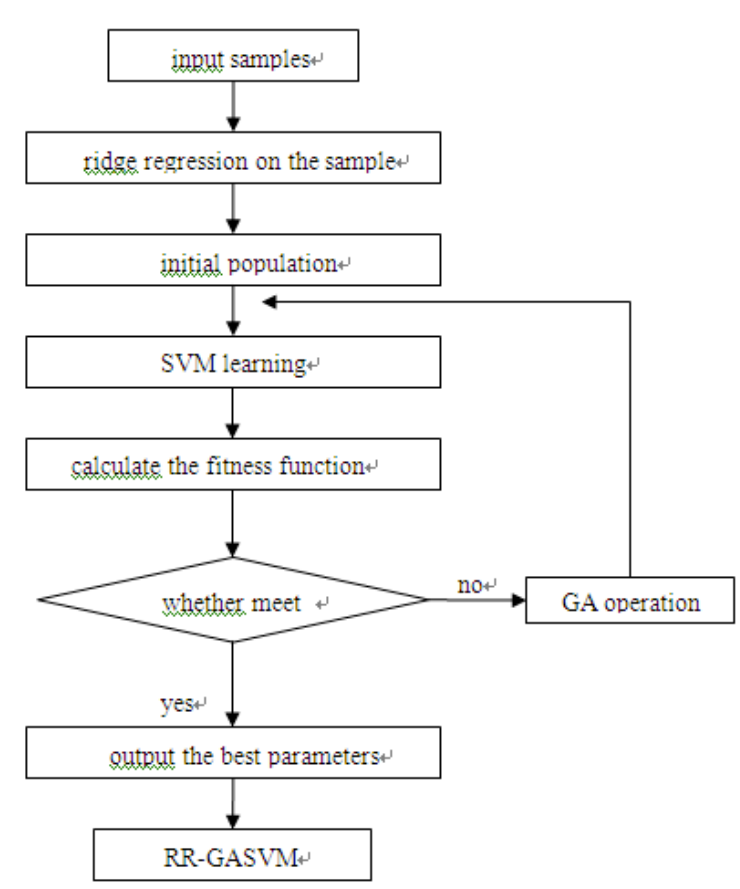

Fig. (3). The flow chart of model optimization of RR-GASVM.

Step 6: Determining whether they meet the final ratio condition, if they meet, we should let them exit the loop, end genetic optimization, and the optimized parameters should be put into the SVM operation, otherwise, go to step 6.

Step 7: Performing crossover operator and mutation operator, and then formation a new generation of individuals, returning to step 3 to continue the implementation of optimization $[13,14]$.

\subsubsection{The Establish of Index System}

The objective comprehensive evaluation index system of land utilization in Zhejianghu must be based on the evaluation index system of data collection. The sample datum is from the "Statistical Yearbook of Anhui in 2013", including the rate of the natural, economic and social aspects. This paper established evaluation index system of comprehensive utilization of land from three aspects of economic, ecological and social benefits on the basis of previous studies. The economic benefit indexes including the GDP of everyone (wanyuan) $X_{1}$, gross value of industrial output (one hundred billion yuan) $X_{2}$, everyone's disposable income of urban(wanyuan) $X_{3}$, everyone's grain yield $(\mathrm{kg}) \mathrm{X}_{4}$. Ecological benefit indexes including everyone's park green space area $\left(\mathrm{m}^{2}\right) \mathrm{X}_{5}$, forest coverage rate $(\%) \mathrm{X}_{6}$, city green coverage rate (\%) $\mathrm{X}_{7}$, nature reserve (\%) $\mathrm{X}_{8}$, the proportion of the available jurisdiction area of everyone (mu) $\mathrm{X}_{9}$ grassland area, per capita afforestation area $(\mathrm{mu}) \mathrm{X}_{10}$. Social benefit indexes including the urban population proportion $(\%) \mathrm{X}_{11}$, the natural population growth rate (\%) $\mathrm{X}_{12}$, the area of established $\mathrm{X}_{13}$ (Thousands of square kilometers), the population density (people/ Thousands of square kilometers) $\mathrm{X}_{14}$, agricultural utilization of land (\%) $\mathrm{X}_{15}$, the family housing area of rural residents $\left(\mathrm{m}^{2}\right) \mathrm{X}_{16}$, everyone's urban road area $\left(\mathrm{m}^{2}\right) \mathrm{X}_{17}$, density of urban population $\mathrm{X}_{18}$ (people/ thousands of square kilometers), the urban population proportion (\%) in the end 
Table 1. The value of ridge regression coefficient when $k$ is different.

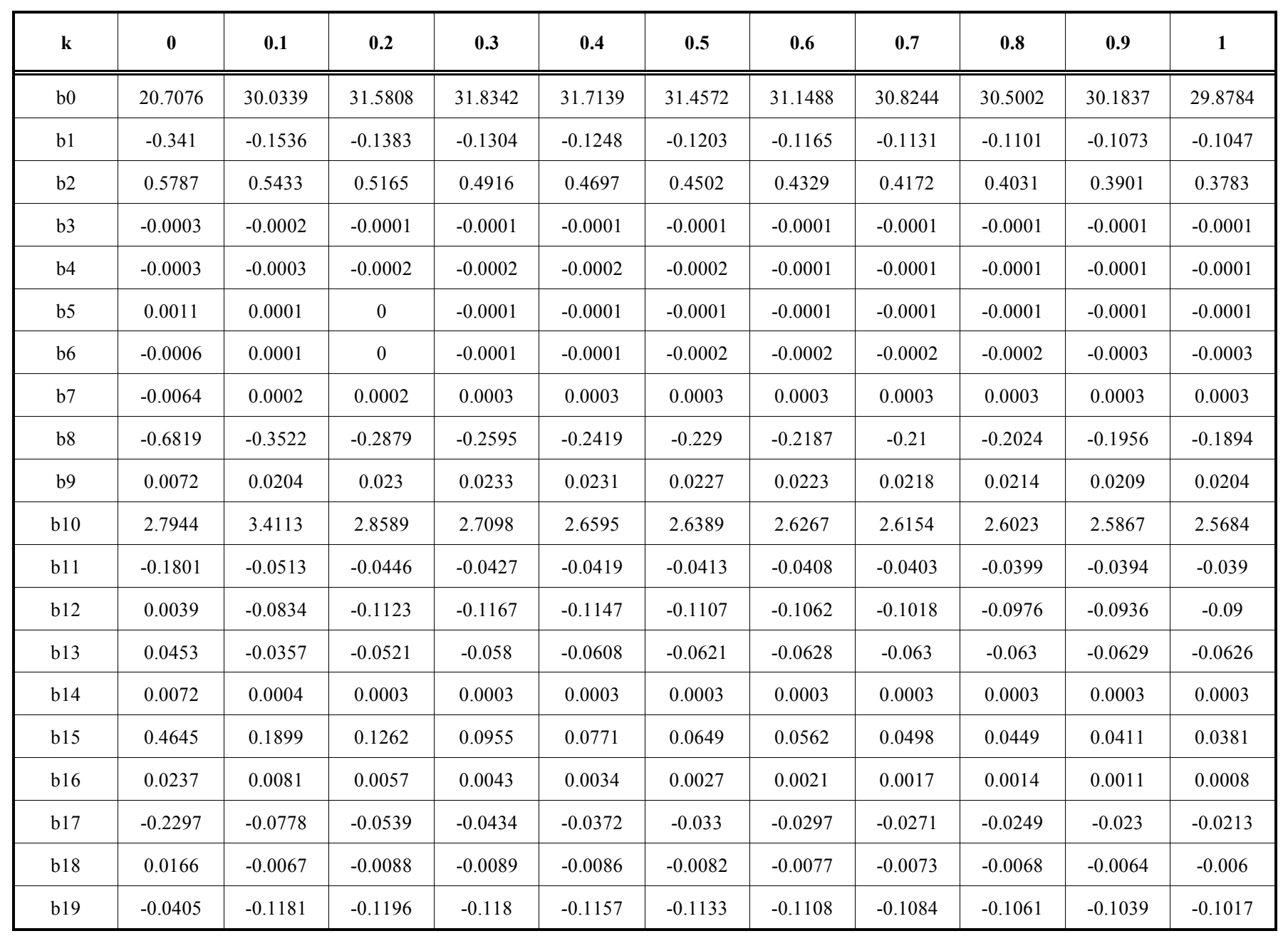

Table 2. GA-SVM and RR-GA-SVM training and testing results.

\begin{tabular}{|c|c|c|c|c|}
\hline Evaluation Method & Training/Test Sets & Mean Square Error MSE & The Correlation Coefficient R & Time \\
\hline \hline GA-SVM & $28 / 29$ & 3.604 & $95.345 \%$ & $166.528 \mathrm{~S}$ \\
\hline RR-GA-SVM & $28 / 29$ & 2.686 & $97.079 \%$ & $158.879 \mathrm{~S}$ \\
\hline
\end{tabular}

of year $X_{19}$. The disposable income of urban one person refers to that part of all cash receipts used to arrange daily life can be reflected, it is income which deducted pay income tax, social-security fees of personal pay as well as the income of the person who was researched after charge to an account. The utilization of agricultural land, the index of agricultural land, refer to the land used for agricultural production directly, including cultivated land, garden land, forest land, grassland and the proportion of other land area accounting for total land area. (Omit table).

\section{RESULTS}

In this paper, data is from China statistical yearbook of 2013, the RR- GASVM of land utilization evaluation in Jiangzhehu. Firstly, use ridge regression to remove linear vari- able existing in the evaluation index system of comprehensive utilization of land and modify appropriately when $\mathrm{k}$ is the value of $0.05,0.1,0.2,0.3,0.4,0.5,0.6,0.7,0.8,0.9,1$, while combining the ridge regression coefficient of different $\mathrm{k}$ values [15], such as Table $\mathbf{1}$.

To get rid of the independent variables of stable standardized ridge regression coefficient and small absolute value, the independent variables of unstable standardized ridge regression coefficient and vibration tending to zero, the independent variables of unstable standardization ridge regression coefficient. Through the analysis of Table 1, compared the ridge trace figure the integrity of evaluation index system of comprehensive utilization of land in economic, ecological and social benefits, the retain indexes is such as urban population proportion $\mathrm{X}_{11}$, the growth rate of natural population $\mathrm{X}_{12}$, 
GDP $X_{1}$, the output of gross industrial value of everyone $\mathrm{X}_{2}$, disposable income urban everyone $\mathrm{X}_{3}$, the established area $X_{13}$, population density $X_{14}$, urban road area of everyone $X_{17}$, urban greening coverage $X_{7}$, available grassland area of everyone $\mathrm{X}_{9}$, planting area of everyone $\mathrm{X}_{10}$. The comprehensive utilization evaluation results of land in Jiangzhehu based on RR - GASVM and GASVM are in the following Table 3.

By comparing the predicted results of GA-SVM and RRGA-SVM from Table 2, to get the following conclusions based on SVM and RR-GASVM.

(1) In terms of precision of land's intensive utilization, the accuracy of RR-GASVM evaluation is higher than that of the standard of the GASVM land evaluation and the evaluation device of RR -GASVM is faster than that of GASVM in evaluation convergence speed. When the quantity of data is larger, it is required for large computing resources solving process of GASVM, which influenced the speed of assessment. Processing and obtain the index of comprehensive land utilization, can improve the accuracy and efficiency of land utilization evaluation by RR GASVM [16].

(2) The data of land intensive utilization can greatly reduce the complexity of the training model by ridge regression, which reduced the model fitting phenomenon and the storage space to a certain extent. It is sped up the training speed of SVM and obtained satisfactory results. Choosing 29 as test samples, 28 as training samples, evaluation accuracy is higher based on RR- GASVM than that of using GASVM, and its training time is short. The mean square error of comprehensive evaluation of land utilization (that is MSE) is 2.68608 , that the time of running is 158.879183 s and correlation coefficient $\mathrm{R}$ is $97.0796 \%$, which shows that the prediction result is scientific. In the meantime, the mean square error of comprehensive evaluation of land utilization (MSE) is 3.60413,that the operation time is $166.528659 \mathrm{~s}$ and correlation coefficient $\mathrm{R}$ is $95.3453 \%$ based on GASVM on the same condition.

(3) The ranking of evaluation of land's comprehensive utilization in Shanghai, Jiangsu, Zhejiang is three, four, and six. The land's comprehensive utilization in above areas is similar to the areas of Beijing, Tianjin, Shandong, Guangdong and Liaoning, and the ranking of these areas are located in the top 10 .

\section{DISCUSSION}

(1) From the effect of comprehensive utilization of land, people can conclude that the ranking of land's comprehensive utilization effect is located at the top in Jiangzhehu and disposable income of one person, grain output of everyone, and the ranking of GDP of everyone is better. Specifically, the ranking of disposable income of everyone in Jiangzhehu is $1,4,2$, and the ranking of grain yield is $5,2,17$. Overall, the ranking is not synchronous, the industrial output of Jiangzhehu ranked 15, 2, 5 and established area ranked 20, 3, 5 respectively.

(2) From the demographic situation, the distribution of population is non-uniform in Jiangzhehu. The proportion of urban population of the 3 areas ranked 1, 6, 5. The urban population at the end of year is seated 1, 6 and 5. Population density ranked 7, 21, 23 respectively. The natural population growth rate located at 1819,25 . Urban population density is situated 7,21, 23 respectively.

(3) From the efficiency of agricultural land utilization, the efficiency of agricultural land utilization is low in Jiangzhehu, specifically, the ranking of agricultural land utilization is $28,24,13$, housing area of rural residents in every family lied in 2, 3, 1, as well as urban road area of everyone ranked 28,2 and 6 respectively.

(4) From the aspects of land ecosystem, the difference is large among Jiangzhehu. Specifically, the parks' greenbelt area of everyone in Jiangzhehu ranked 28, 8, 9. The rate of forest coverage ranked $26,23,3$. The coverage rate of city green ranked 18, 3, 11. The population of nature reserve area accounting for total area ranked20, 24, 28. The available grassland area of everyone ranked 28, 27, 22. Forestation area of everyone ranked $28,26,24$ respectively.

\section{SUGGESTIONS}

\subsection{Consolidating the Gained Results of Land Compre- hensive Utilization and Strengthen Innovation of Land Management System}

Jiangzhehu will increase urban construction land directly by promoting the rural collective construction land in market, accelerating rural land circulation equal to the farmers transfer their using right of land, which letting the scattered land together in order to realize intensive and large-scale operation, improving the utilization efficiency of rural land, optimizing allocation of agricultural resources, speeding up agricultural modernization development, improving the efficiency of land utilization, saving the urban construction land.

\subsection{Increasing the Protection of the Ecological Function to Protected Areas}

Jiangzhehu should increase the proportion of nature reserves and the planting area of everyone. In terms of natural environment, these areas should pay attention to the use of pesticides reasonably, control the discharge of industrial waste water, waste gas, focus on the garbage disposal and the level of green, in addition, these areas should control the speed of urban expansion, control the growth rate of natural population, maintain reasonable population density, increase investment in forestry development, and promote the rate of forest coverage, push green tree planting project vigorously, strengthen the construction of city country parks, etc, promote the protection of the ecological function protected areas.

\subsection{Insisting on the Principle of Paying Equal Attention to Development and Consolidation, While the Land is Suitable for Farm, and Plant Forest While the Land is Suitable for Plant}

Jiangzhehu should improve land utilization and the ecological environment at the same time that these areas cultivated land's ability of resisting natural disasters. According to the present situation of farm land in these three which include Jiangsu, Zhejiang and Shanghai, these areas should adjust and optimize industrial layout actively, and promote the synchronous implementation of flat land and land circu- 
lation vigorously, as well as develop the consolidation project of land scientifically and systematically. There is a clear need to supervise the construction of land strictly in the development process of cultivated land occupied, improve the fertility of the land resource by developing constantly as well as reduce water loss and soil erosion, improve the comprehensive ability of resistance to natural disasters and promote land reclamation and ecological restoration as much as possible.

\section{CONCLUSION}

This paper established evaluation index system of the development model of new urbanization.

RR and GA have already widely applied to optimizing calculating at present, intelligent design, neural network training etc., it is a rising method. To establish RR-GA SVM models for comprehensive utilization of land of Jiangzhehu. The result of SVM algorithm trained by RR - GA is better than that of the GA. The study on one group of algorithms of RR - GA SVM, whether in the theory or in practicing, is developing. The existing research results are still quite scattered. Many unknown fields need studying, especially the mathematical foundation of RR - GA SVM seems much weaker, lacks deep theories analysis with general significance, and this is also an important direction that needs to be studied in the future.

\section{CONFLICT OF INTEREST}

The author confirms that this article content has no conflict of interest.

\section{ACKNOWLEDGEMENTS}

This work is supported by Study of new urbanization evaluation based on the intelligent integrated optimization of SVM parameters (2015 Natural science fund project in Anhui province; number: 1508085MG144), and Intelligence improved method of support vector machine and its application in the evaluation of new urbanization (2014 Anhui universities colleges and universities in Anhui province natural science key research project; number: KJ2014A042).

\section{REFERENCES}

Y. Wang and X. Y. Yang, "Study on the Intensive Use of Land in the Process of Urbanization", Journal of Hubei University for
Nationalities (Natural Science Edition), vol. 3, pp. 357-360, 2009.

[2] F. Yang, "Study of intensive use of urban land during the process of urbanization”, Enterprise Economy, vol. 1, pp. 151-154, 2013.

[3] L. P. Qu and L. Q. Zhang, "Reasons and countermeasures for intensive use restriction of urban land", Scientific and Technological Management of Land and Resources, vol. 5, pp. 134-139, 2008.

[4] A.M. Wang, "Problems and dilemmas: review on the study of intensive use of urban land", Journal of Chongqing University (Social Science Edition), vol. 4, pp. 7-10, 2010.

[5] L. Qiu, H. P. Liao, Y. J. Long, and X. H. Li, "The evaluation of urban land intensive utilization and the analysis of the spatiatemporal features in cheng-yu economy zone", Journal of Southwest China Normal University (Natural Science Edition), vol. 1, pp. 208-213, 2010.

[6] W. G. Wang, X. X. Pang, Y. X. Song, and X. H. Chen, "Study on regional problem of land use and function replacement between regions", China Population, Resources and Environment, vol. 9, pp. 68-75, 2012.

[7] H. Li, J. C. Xu, C. Z. Li and Z. Y. Xu, "Path analysis on the impacts of ecological land allocation on land intensive use:a case of the development zones in zhejiang province", China Land Science, vol. 9, pp. 42-47, 2011.

[8] J. Wu, X. H. Deng and P. H. Liu, "Research on evaluation of intensive urban land use based on ahp__ case study of fuzhou education park", Journal of East China Institute of Technology (Social Science), vol. 3, pp. 356-361, 2013.

[9] M. H. He, F. G. Liu, Z. X. Tang and Q. Zhou, "Research on the intensive use of urban land in xining city", Journal of Arid Land Resources and Environment, vol. 3, pp. 44-48, 2014.

[10] D. M. Hawkins and X. R. Yin, "A faster algorithm for ridge regression of reduced rank data", Computational Statistics \&Data Analysis, vol. 40, pp. 253-262, 2002.

[11] Y. Yao, "The Credit Scoring System Based On Support Vector Machine", Journal of System Simulation, vol. 16. pp. 783-787, 2006.

[12] O. L. Mangasarian, "Generalized support vector machines". In A Smola, P. Bartlett, B. SchAolkopf, and D. Schuurmans, editors, Advances in Large Margin Classifiers, Cambridge, MA, pp. 135146,2000

[13] F. E. H. Tay and L. J. Cao. "Modified support vector machines in financial time series forecasting", Neurocomputing, vol. 48, pp. $847-861,2002$.

[14] K. M. Mehmet, "Multi-objective genetic algorithm for motif discovery", Expert Systems with Applications, vol. 36, pp. 10391047, 2007.

[15] H. M. Jae and Y. C. Lee, "Bankruptcy prediction using support vector machine with optimal choice of kernel function parameters", Expert Systems with Application, vol. 28, pp. 603-614, 2005.

[16] P. F. Pai and W. C. Hong, "Forecasting regional electricity load based on recurrent support vector machines with genetic algorithms", Electric Power Systems Research, vol. 45, pp. 417-425, 2005. 